\title{
Opinion mining on newspaper headlines using SVM and NLP
}

\author{
Chaudhary Jashubhai Rameshbhai, Joy Paulose \\ Dept. of Computer Science, Christ University, India
}

\begin{tabular}{|c|c|}
\hline Article Info & ABSTRACT \\
\hline $\begin{array}{l}\text { Article history: } \\
\text { Received Jan 4, } 2018 \\
\text { Revised Jul 23, } 2018 \\
\text { Accepted Dec } 15,2018\end{array}$ & $\begin{array}{l}\text { Opinion Mining also known as Sentiment Analysis, is a technique or procedure which } \\
\text { uses Natural Language processing (NLP) to classify the outcome from text. There are } \\
\text { various NLP tools available which are used for processing text data. Multiple research } \\
\text { have been done in opinion mining for online blogs, Twitter, Facebook etc. This pa- } \\
\text { per proposes a new opinion mining technique using Support Vector Machine (SVM) }\end{array}$ \\
\hline $\begin{array}{l}\text { Keywords: } \\
\text { Newspaper } \\
\text { Sentiment analysis } \\
\text { Opinion mining } \\
\text { NLTK }\end{array}$ & $\begin{array}{l}\text { and NLP tools on newspaper headlines. Relative words are generated using Stan- } \\
\text { ford CoreNLP, which is passed to SVM using count vectorizer. On comparing three } \\
\text { models using confusion matrix, results indicate that Tf-idf and Linear SVM provides } \\
\text { better accuracy for smaller dataset. While for larger dataset, SGD and linear SVM } \\
\text { model outperform other models. }\end{array}$ \\
\hline
\end{tabular}

NLTK

Stanford coreNLPm

SVM

Copyright (C) 2019 Institute of Advanced Engineering and Science.

SGDClassifier

All rights reserved.

Tf-idf

CountVectorizer

\section{Corresponding Author:}

Chaudhary Jashubhai Rameshbhai,

Department of Computer Science,

Christ University Hosur Road, Bangalore, Karnataka, India 560029.

Phone: +91 7405497405

Email: chaudhary.rameshbhai@cs.christuniversity.in

\section{INTRODUCTION}

Opinion Mining or Sentiment Analysis is a task to analyze opinions or sentiments from textual data. It is useful in analyzing NLP applications. With the development of applications like network public opinion analysis, the demand on sentiment analysis and opinion mining is growing. In today's world, utmost people are using internet and social media platforms, to share their views. These analysis are available in various forms on the internet, like reviews about product, Facebook post as feedback, Twitter feeds, blogs etc. At present, news play a dynamic role in developing a person's visions and opinions related to any product, political party or company. News article published in the newspaper or shared on the web can sometimes create negative or positive impacts on the society on large scale. As per Dor (1), most of the people judge the news contents directly by scanning only the news headlines relatively than going through the complete story. Hence, minor headlines can also impact on large scale. In this paper, Opinion mining is performed based on just the headlines without going through whole articles. The proposed method begins with data collection and preprocessing. Data are collected from different news source using Python newspaper package. Review for news headlines are assigned either +1 or -1 manually based on sentiments. In order to perform SVM to build a classification model, news headline data are fetched and processed in CoreNLP (2). CoreNLP returns set of relative words which are imported into count vectorizer (3) to generate matrix.

This paper is organized as follows: Section 2 provides overview of related works on opinion mining. Section 3 contains elaborate explanation of the proposed method. Section 4 discusses the experimental results of three models. Section 5 concludes and provides future scope of this proposed method. 


\section{RELATED WORK}

Agarwal et al. (4) proposed a method containing two algorithms. First algorithm is used for data pre-processing while other to detect polarity value of a word. Natural Language Processing Tool (NLTK) and SentiWordNet are employed to build the proposed method. NLTK is a Python library for word tokenization , POS (Part of Speech) - Tagging, Lemmatization and Stemming. SentiWordNet (5) lexicon, a method extended from WordNet is specifically designed for Sentiment Analysis. Each word is assigned with positive or negative numerical scores. Negative words are denoted with negative score, while positive words are assigned with positive score. Output of NLTK is fed to SentiWordNet in order to assign numerical score for each word and compute the final sentiment score, which is sum of all the numerical scores. If the final value is greater or equal to 0 , the headline is classified as positive or negative headline.

Yang et al. (6) proposed a hybrid model for analyzing sentiments of textual data for a single domain. It is implemented domain wise due to increase in complexity upon segregation. Single classification model is used to segregate the responses as positive, negative and neutral. The model is a combination of multiple single classification method which provides more efficient classification.

Rana and Singh (7) compared two machine learning algorithms Linear SVM and Naive Bayes for Sentiment Analysis. Review on movies are used as dataset containing 1000 samples. Proter Stemmer is employed to preprocess dataset. While Rapid Miner tool is used to generate model, Linear SVM and Naive Bayes are used as classifier. Precision, recall and accuracy of both the models are calculated. From the result, it is observed that the Linear SVM gives better result than Naive Bayes.

Bakshi et al. (8) proposed an approach to classify positive, negative and neutral tweets of Twitter. It is focused on a single company Samsung Electronics Ltd. Data is fed and processed to clean the data. The algorithm is applied to analyze the sentiment of tweets and segregate into different categories.

Aroju et al. (9) proposed a method to perform Opinion Mining on three different newspapers related to similar news. SVM and Naive Bayes are used for opinion mining. Around 105 news headlines are collected from three different sources (35 headlines from The Hindu, The Times of India and Deccan Chronicle Newspaper). Data is processed using POS - tagger and stemming. Weka tool is used to implement the method. For experimental results, F-score, Precision and Recall are calculated. From the result it is evident that The Hindu newspaper contains more positive news than The Times of India and Deccan Chronicle.

Hasan et al. (10) proposed an algorithm which uses Naive Bayes to perform opinion mining. Data are reviewed in English from e-commerce website Amazon. The reviews are also translated to Bangla using Google Translator. Opinion mining is calculated for reviews in both Bangla and English. The Bangla dataset translated from English contains noise. The reviews in Bangla as training data are fed into Naive Bayes to build classifier excluding noisy words.

Akkineni et al. (11) proposed a method of classifying opinion, opinions are classified based on subject of opinions and objective of having such opinion, this method helps to classify whether sentence is a fact or opinion. Approach adopted for classifying in this paper range are heuristic approach where results within a realistic time-frame. They are likely to produce the results themselves but are mostly used with the optimized algorithms, Discourse Structure which focuses on the given text that just communicates a message, and linking it to how that message constructs a social reality or view of the world,key word analysis which classifies text by affect categories based on the presence of unambiguous affect words such as happy, sad, afraid, and bored, Concept analysis which concentrates on semantic analysis of text through the use of web ontologies or semantic networks. The conceptual and affective information associated with natural language opinions are aggregated.

Arora et al. (12) proposed Cross_BOMEST, a cross domain sentimental classification. Existing method BOMEST, it retrieves +ve words from a content, followed by determination of +ve word with assistance of Ms Word Introp. In order to escalate the polarity it replaces all it's synonym. Moreover, it helps in blending two different domains and detect self-sufficient words. The proposed method is test and implemented on Amazon dataset. Total of 1500 product reviews are randomly selected for both +ve and -ve polarity. Out of which 1000 are used for training and remaining to test the classification model. As a result, when applying on cross domain precision, accuracy of $92 \%$ is achieved. For single domain, precision and recall of BOMEST is improved by $16 \%$ and $7 \%$. Thus, Cross_BOMEST improves the precision and accuracy by $5 \%$ when compared to other existing techniques.

Susanti et al. (13) employs Multinomial Naïve Bayes Tree which is combination of Multinomial Naïve Bayes and Decision Tree. The technique is used in data mining for classification of raw data. Multinomial Naïve Bayes method is used specifically to address frequency calculation in the text of the sentence or docu- 
ment. Documents used in this study are comments of Twitter users on the GSM telecommunications provider in Indonesia.[] This paper used the method to categorize customers sentiment opinion towards telecommunication providers in Indonesia. Sentiment analysis only consists of positive, negative and neutral class. Decision Tree is generated with this method and roots in the feature "aktif", where probability of feature "aktif" belongs in positive class. In result and analysis, it is indicated that the highest accuracy of classification using Multinomial Naïve Bayes Tree (MNBTree) method is $16.26 \%$ when using 145 features. Furthermore, the Multinomial Naïve Bayes (MNB) yields the highest accuracy of $73,15 \%$ by using all dataset of 1665 features.

In this type of research selection of appropriate feature is one of the challenges. Many researchers are using decision tree and n-gram approach for feature selection and Supervised Machine learning technique for model building. The tedious job in this type of research is data preprocessing. Most of the researchers are using NLP tools for data preprocessing (14), (15), (16), (17), (18). In this paper, n-gram and coreNLP is used for feature selection and Linear SVM are used for model building.

\section{PROPOSED WORK}

Many algorithms are available for finding sentiment from text, but they are performed on large text dataset like movie reviews, product reviews etc. Finding opinions from news headlines is also possible but the accuracy of existing algorithm is not satisfactory. This paper tries to improve the accuracy of existing algorithm (4) using different approach. The proposed method is distributed into three processes.

As shown in Figure 1, Process I is regarding data collection and pre-processing. Process II is the core fragment to build classifier and Process III tests the classification model on test data. These processes are discussed in detail further.

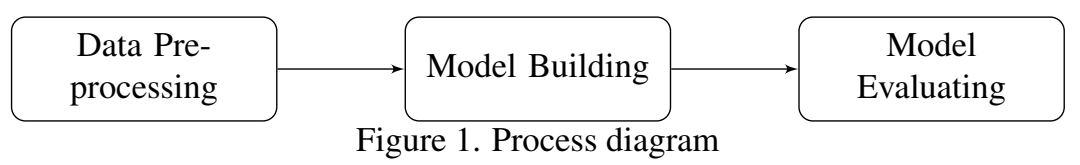

\subsection{Data Pre-processing And Model Building}

In this process, data pre-processing and model building are implemented. Figure 2 depicts the basic flowchart of the data preprocessing and model building. Stop words are removed from news headlines followed by converting uppercase texts to lowercase. The semi-processed headlines are fed to coreNLP. The output of coreNLP with sentiment scores are set as input for process II. The input is received from process I and converted into unigram and bi-gram representation. Model A is generated from the unigram and bi-gram representation. Model A employs Linear SVM. Data representation is further converted into Tf-idf resulting in Model B and C. Model B and C employ Linear SVM. However, unlike model B, model C uses SGD classifer to train the data.

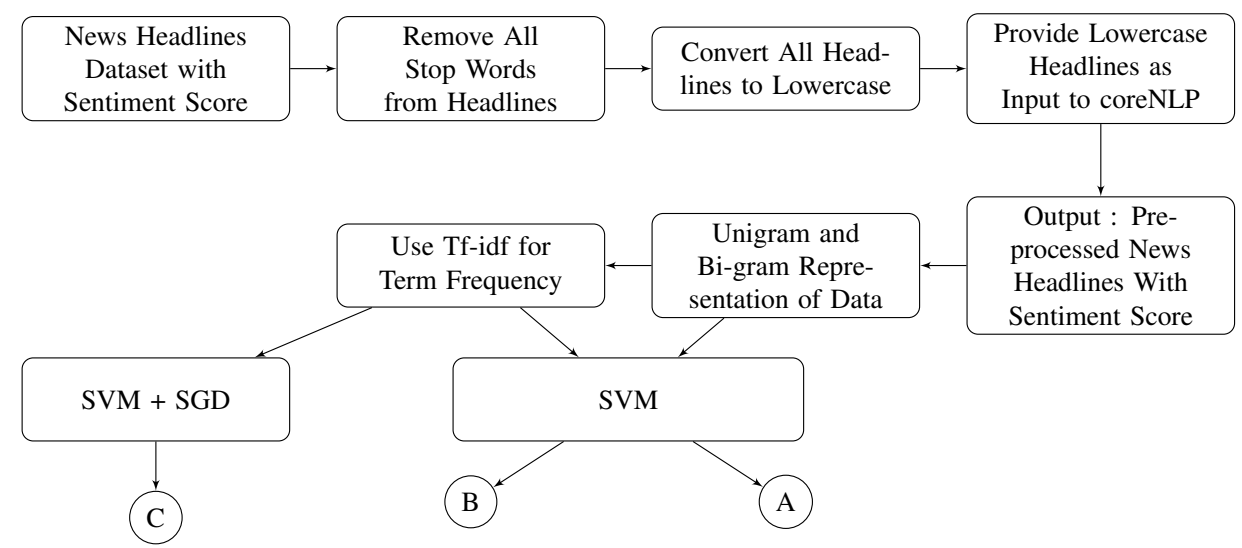

Figure 2. Flow diagram of data pre-processing and model building 
Data are collected from the website http://www.indianexpress.com during August 2017. Figure 3 depicts sample unprocessed data. 1472 news headlines are collected and manually classified as either +1 or -1 . For positive news, sentiment score is +1 , while for negative news is -1 . Subsequently, after allocating the sentiment score, all the stop words from headlines such as: is, the, are etc are eliminated. NLTK STOP WORDS is used to perform the task of removing stop words. The algorithm is case sensitive for which all the headlines are converted to lowercase. The data is fed to Stanford CoreNLP to generate the dependency parser. Standford CoreNLP dependency parser (19) checks the grammatical construction of a sentence, which establishes relation between "ROOT" word and altering words. To understand how coreNLP works consider news headlines: "Two killed in car bomb in Iraq Kirkuk". Figure 4 depicts parsing of sample data using dependency parser. "ROOT" word of the headline is returned and relation between each word of the headline. From the sample data, killed is returned as "ROOT" word and relation between all the words. Figure 5 shows parsing of sample headline using dependency parser and converting the output in the form of string array. String array consists of "ROOT" word and relative words. The process is applied on all the data to generate array of strings with "ROOT" word and relative words. Figure 6 is statistical representation of frequency of words in data. Figure 7 depicts sample data with sentiment score. The processed data are input for process II.

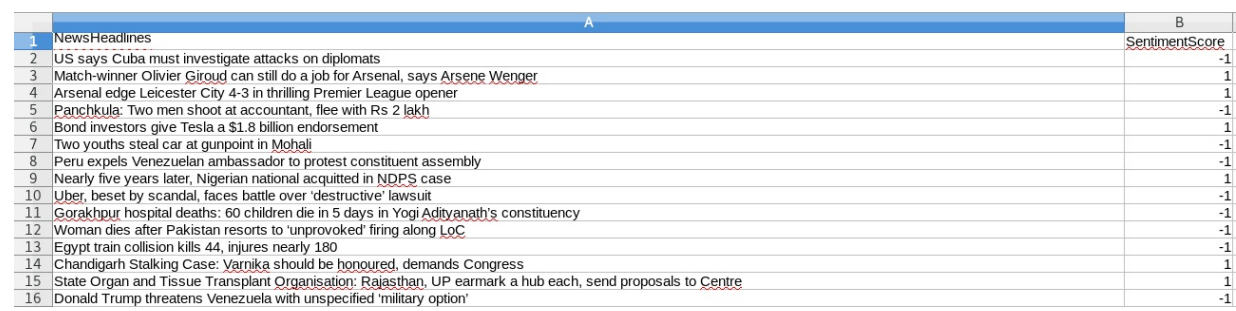

Figure 3. Dataset screenshot

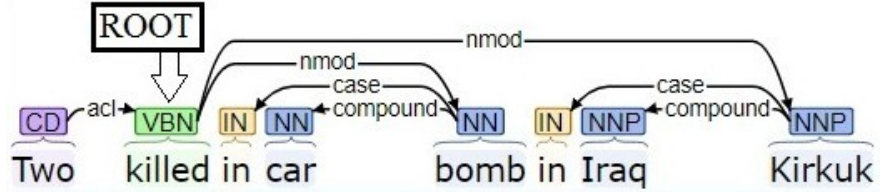

Figure 4. Dependency parser working diagram

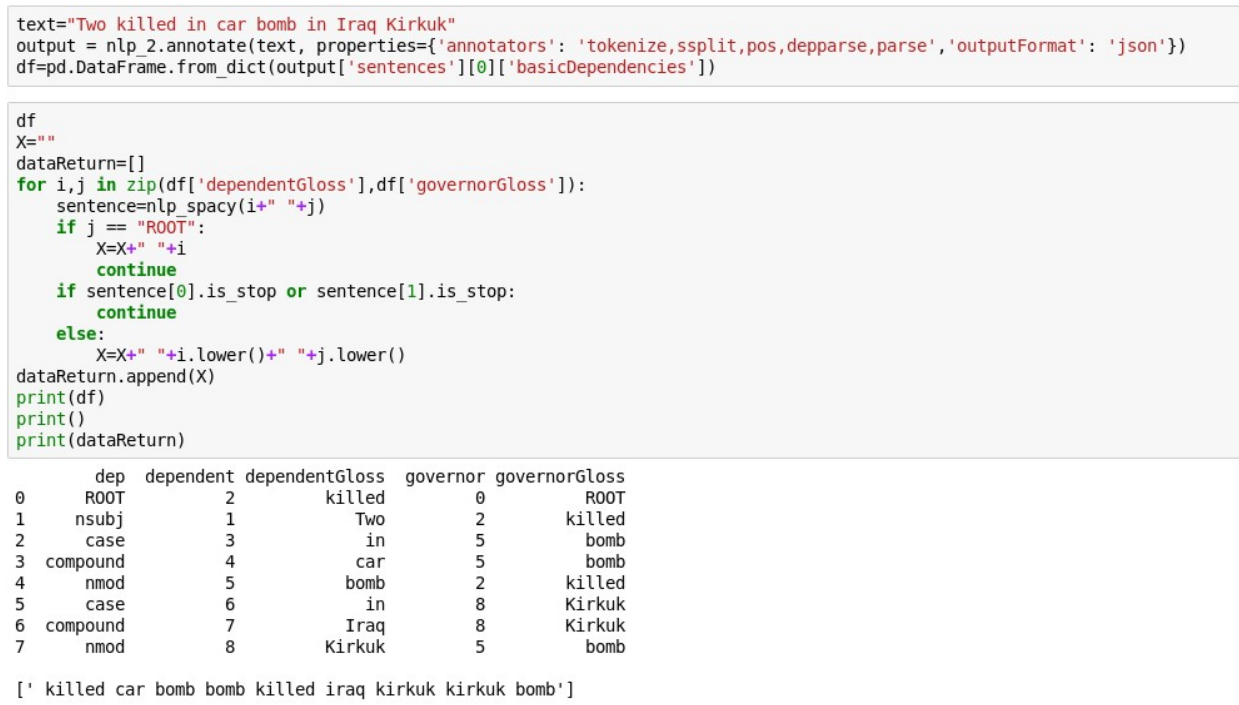

Figure 5. Generating pandas dataframe using coreNLP 


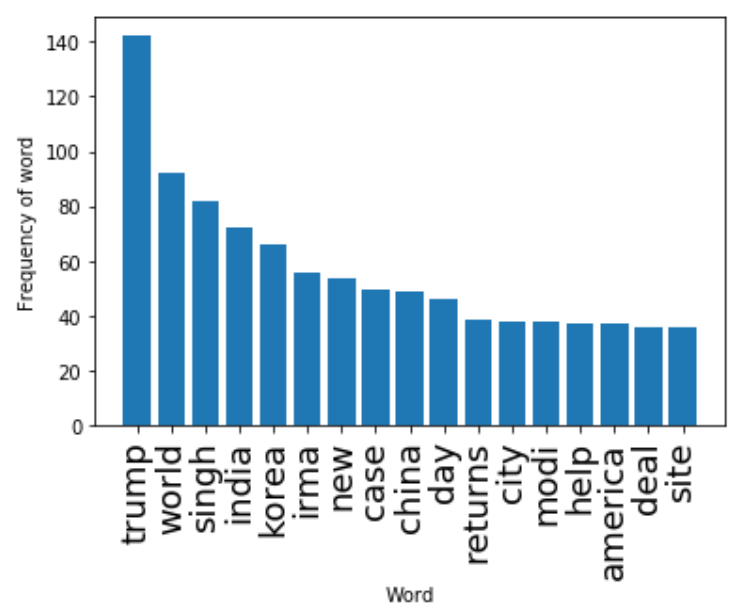

Figure 6. Most frequently occurred words in dataset

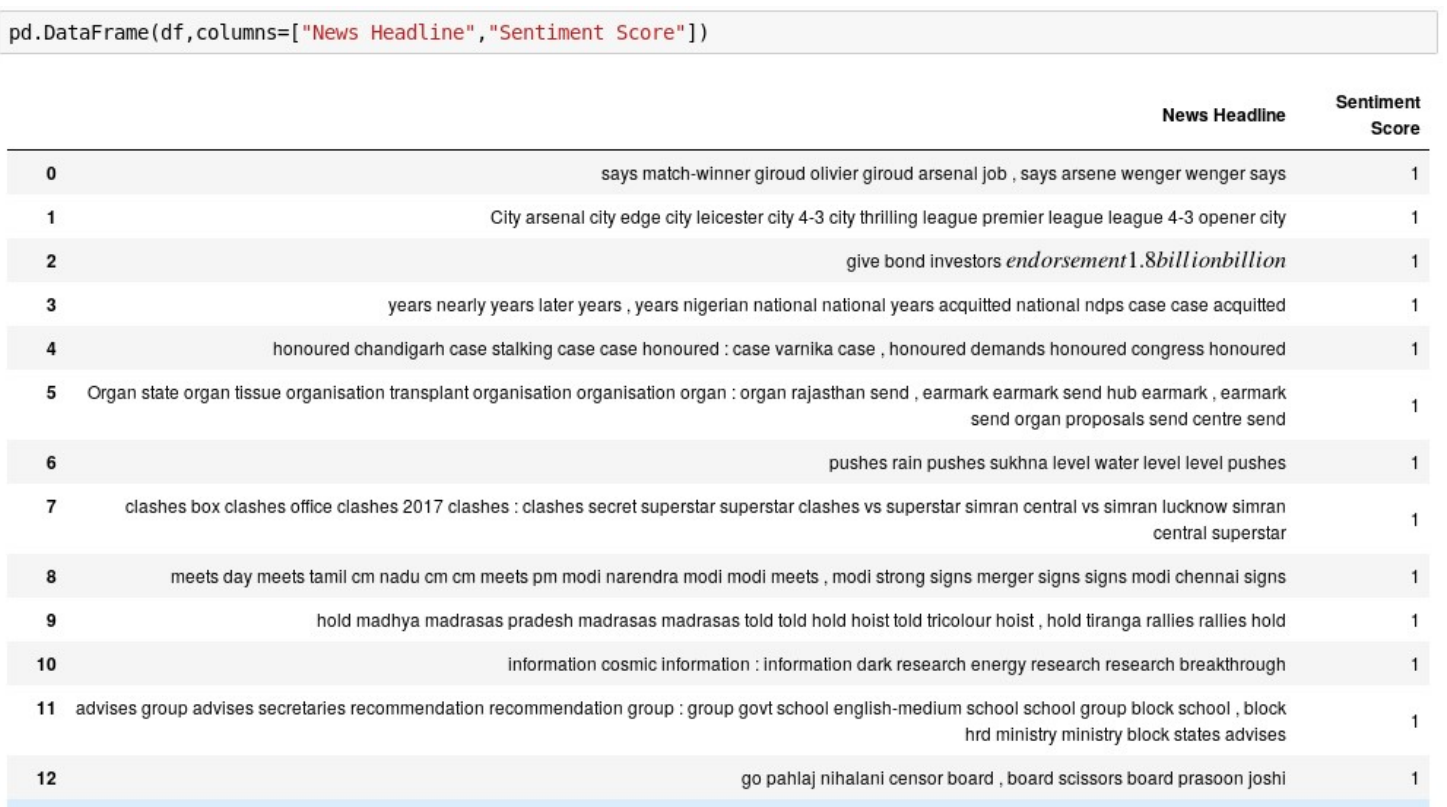

Figure 7. Sample dataset after applying coreNLP

\subsection{Unigram and Bi-gram Representation of Data}

Before building the model, the raw data is converted from string to numerical values. In machine learning, Text Analysis is a key application area. Most of the algorithms accept numerical data with fixed size rather than text data of varying size. A collective approach uses a document-term vector where individual document is encrypted as a discrete vector that sums occurrences for each word in the vocabulary it contains (3). For example, consider two one-sentence documents:

D1: "I like Google Machine Learning course"

D2: "Machine Learning is awesome"

The vocabulary V $=\{\mathrm{I}$, like, Google, Machine, Learning, course, is, awesome $\}$ and two documents can be encoded as v1 and v2. Figure 8 and 9 show the representation of given sentences in unigram and bi-gram model. The bi-gram model refines data representation where occurrences are determined by a sequence of two words rather than individually. 


\begin{tabular}{|l|l|l|l|l|l|l|l|l|}
\hline & I & Like & Google & Machine & Learning & Course & Is & Awesome \\
\hline v1 & 1 & 1 & 1 & 1 & 1 & 1 & 0 & 0 \\
\hline v2 & 0 & 0 & 0 & 1 & 1 & 0 & 1 & 1 \\
\hline
\end{tabular}

Figure 8. Unigram representation of given vocabulary

\begin{tabular}{|l|l|l|l|l|l|l|}
\hline & I like & Like Google & Google Machine & Machine Learning & $\ldots \ldots$. & Is awesome \\
\hline v1 & 1 & 1 & 1 & 1 & $\ldots \ldots$ & 0 \\
\hline v2 & 0 & 0 & 0 & 1 & $\ldots \ldots$ & 1 \\
\hline
\end{tabular}

Figure 9. Bi-gram representation of given vocabulary

Figure 10 shows snipped code to generate unigram and bi-gram representation of given data. Data is an array of 4 news headlines. Here, CountVectorizer () is used to convert string data into numeric values. To generate unigram model, pass argument ngram_range $=(1,1)$ and ngram_range $=(1,2)$ for bi-gram. In figure, there are two matrices generated using pandas library. In matrix, columns represents unique words (31 for unigram and 61 for bi-gram) and rows represents 4 news headlines. If the word exists in particular news headlines the value for that particular feature will be 1 and if the word exists twice then the value for that feature will be 2. Value depends on frequancy of word in headline. This method is used when a model is built in Process II.

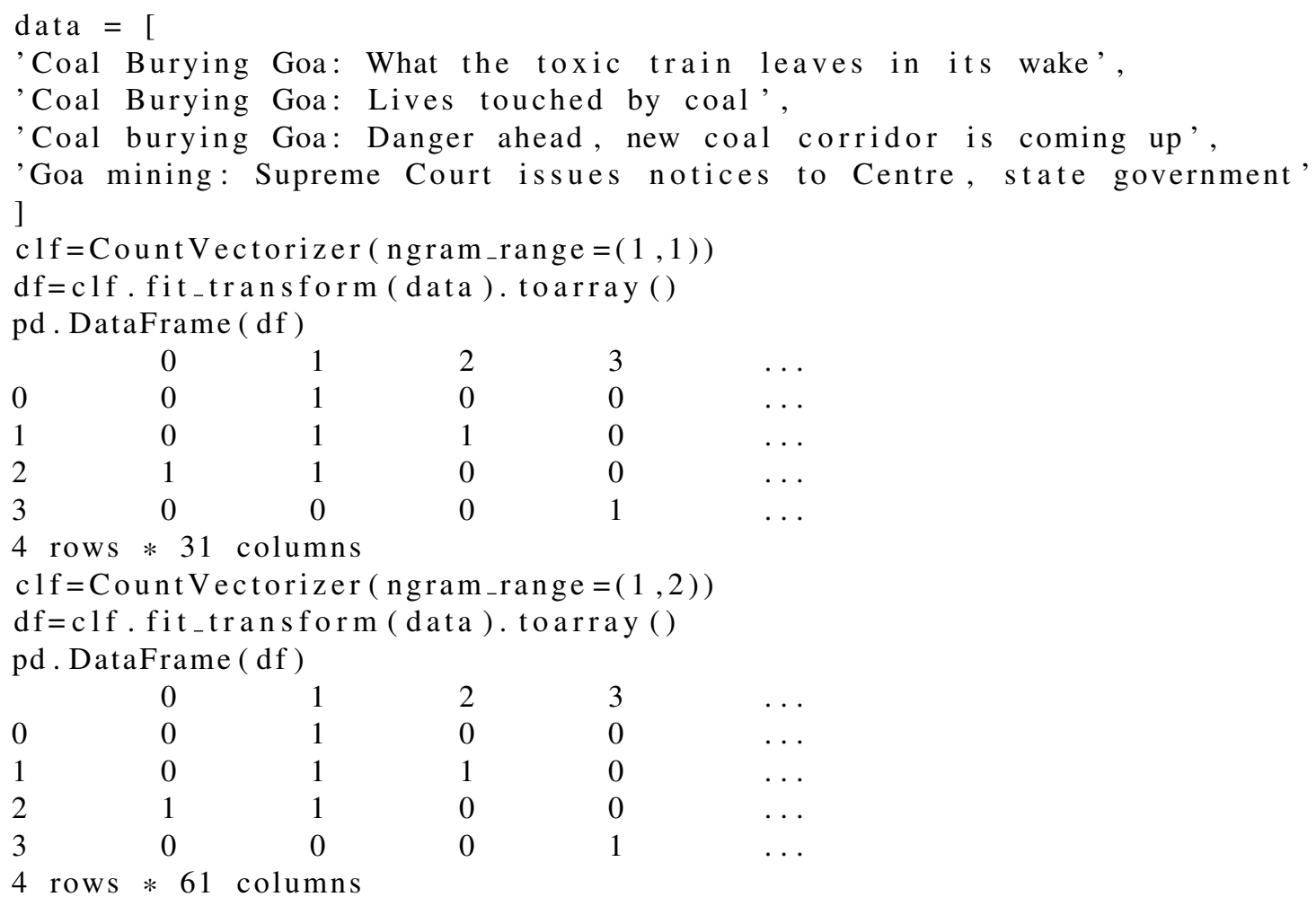

Figure 10. Snipped python code to generate Unigram and Bi-gram from given string data. 


\subsection{Model A. Linear SVM}

In this process, Linear SVM is used to build the model and the data that has been used to build the model is Numeric. The Description for total dataset is shown in Table 1. The Matrices in Figure 11 and 12 have been generated by using unigram and bi-gram, column shows the number of words in headlines and row represents number of headlines. When the value in Matrix is 0, it means that particular word does not exist in the headlines. In unigram, number of feature depends on total unique words in dataset.

Table 1. Dataset Description

\begin{tabular}{|c|c|c|}
\hline & Total Sample & Total Feature \\
\hline Unigram & 1472 & 4497 \\
\hline Bi-gram & 1472 & 13832 \\
\hline
\end{tabular}

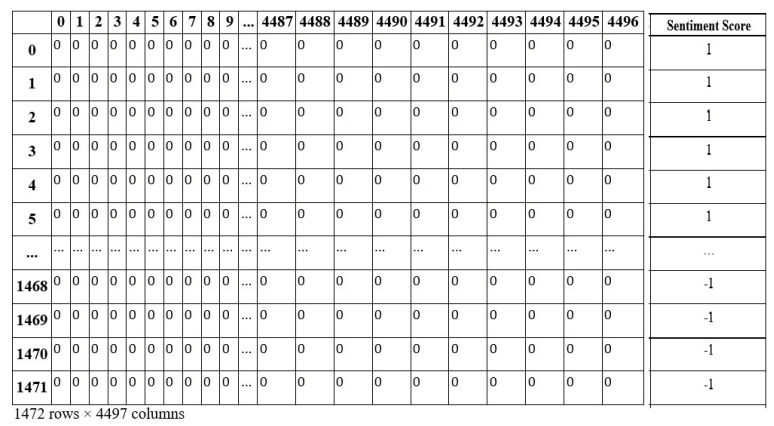

Figure 11. Dataset representation in Unigram model.

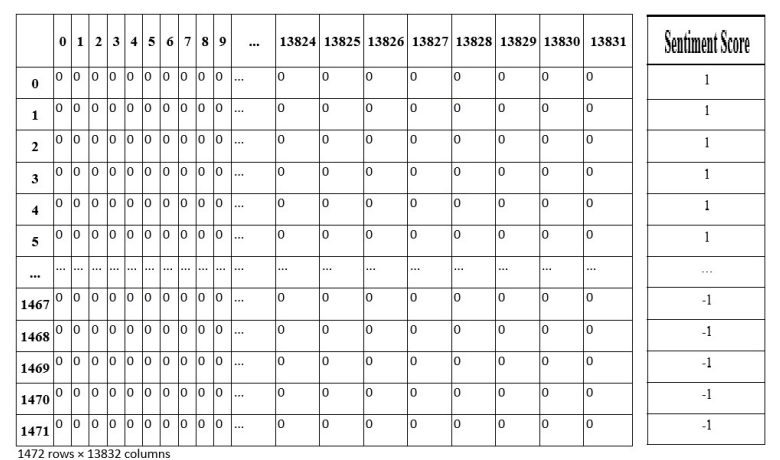

Figure 12. Dataset representation in Bi-gram model.

Table 1 shows total number of features and samples in unigram and bi-gram. Total sample size is total number of news headlines and total feature size is number of unique words in dataset. Here, total sample dataset size is same for both models. But in unigram model total number of feature is 4497 and 13832 for bi-gram model. For building this model, $80 \%$ data are considered for training set and $20 \%$ are considered for evaluating the model. Here, kernel is linear because we have two class labels, so SVM generates linear hyper plane which will separate words. It separates all negative news headline words and positive news headline words.

\subsection{Model B. Tf-idf and Linear SVM}

Linear SVM is used in this model building and the dataset is converted into document frequency using Tf-idf. Tf is Term-frequency while Tf-idf (3) is Term-frequency time's inverse document-frequency. It is used to classify the documents. The main aim of Tf-idf is to calculate the importance of a word in any given headline with respect to overall occurrence of that word in the dataset. The importance of a word is high if it is frequent in the headline, but less frequent in overall headline. Tf-idf can calculated as follows (3):

$$
t f i d f(t, d)=t f(t, d) * i d f(t)
$$


Where $t f(t, d)$ is term frequency in particular headline, the term occurred number of times in particular headline and is multiplied with $\operatorname{idf}(\mathrm{t})$.

$$
i d f(t)=1+\log \frac{1+n_{d}}{1+d f(d, t)}
$$

Where $n_{d}$ is the total number of headlines, and $\mathrm{df}(\mathrm{d}, \mathrm{t})$ is the number of headlines that contain term $\mathrm{t}$. The resulting Tf-idf vectors are then normalized by the Euclidean norm:

$$
v_{n o r m}=\frac{v}{\|v\|^{2}}=\frac{v}{\sqrt{v_{1}^{2}+v_{2}^{2}+. .+v_{n}^{2}}}
$$

$$
\begin{aligned}
& \text { data_counts }=\left[\left[\begin{array}{lll}
3, & 0, & 1
\end{array}\right],\right. \\
& {[2,0,0] \text {, }} \\
& {[3,0,0] \text {, }} \\
& {[4,0,0] \text {, }} \\
& {[3,2,0] \text {, }} \\
& [3,0,2]]
\end{aligned}
$$

For example, Tf-idf is computed for the first term in the first document in the data_counts array as follows:

To calculate Tf-idf of first term in document:

Total No. of Documents : $n_{d, t e r m 1}=6$

Total No of Documents which contain this term 1 :

$$
d f(d, t)_{\text {term } 1}=6
$$

idf is for term 1 :

$$
\begin{gathered}
i d f(d, t)_{\text {term } 1}=1+\log \frac{n_{d}}{d f(d, t)}=\log \frac{6}{6}+1=1 \\
t f i d f_{\text {term } 1}=t f_{\text {term } 1} * i d f_{\text {term } 1}=3 * 1=3
\end{gathered}
$$

Similarly for other two terms:

$$
\begin{gathered}
t f i d f_{\text {term } 2}=0 *\left(\frac{6}{1}+1\right)=0 \\
t f i d f_{\text {term } 3}=0 *\left(\frac{6}{1}+1\right)=2.0986
\end{gathered}
$$

Represent Tf-idf in vector:

$$
\text { tfidf } f_{\text {raw }}=[3,0,2.0986]
$$

After applying Euclidean norm:

$$
\frac{[3,0,2.0986]}{\sqrt{\left(3^{2}+0^{2}+2.0986^{2}\right)}}=[0.819,0,0.573]
$$


In $\operatorname{idf}(t)$, to avoid zero divisions "smooth_idf=True" adds " 1 " to the numerator and denominator.After modification in equation the first two term value will be same but in term 3 value changes to 1.8473:

$$
t f i d f_{\text {term } 3}=1 * \log \frac{7}{3}+1=1.8473
$$

$$
\frac{[3,0,1.8473]}{\sqrt{\left(3^{2}+0^{2}+1.8473^{2}\right)}}=[0.8515,0,0.5243]
$$

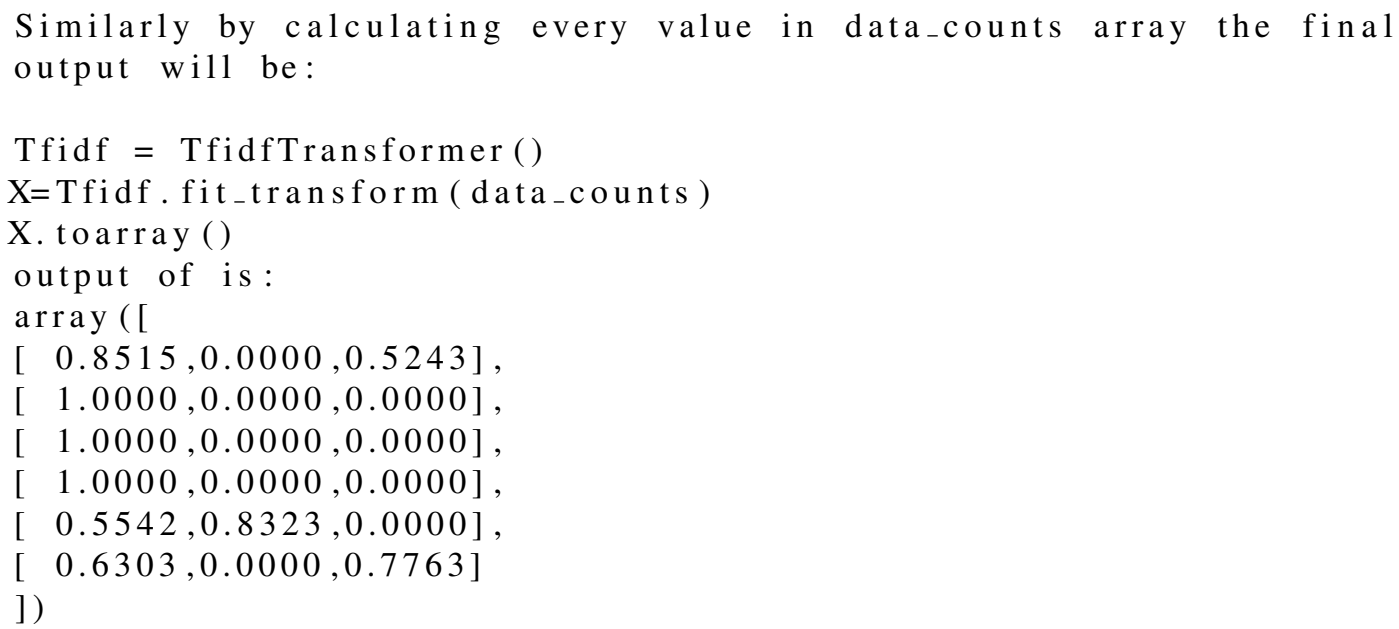

The total number of sample data size and feature data size will remain same for building this model. Here, the frequency of each word is changed according to Tf-idf. Unlike the previous model words with less frequency in headlines will have lower values. It means words with lesser frequency will have lesser impact on model. For training, this model is using $80 \%$ data and for testing it is using $20 \%$ data.

\subsection{Model C. Stochastic Gradient Descent (SGD) Classifier}

The SGD is used to train the data for Linear SVM. SGD (3) is a discriminative learning of linear classifiers like SVM and Logistic Regression which is simple and very efficient. SGD has been effectively implemented in numeric data machine learning problems majorly involved in text categorization and NLP. Data provided is in sparse, the classifiers in SGD efficiently scales to the problem with more than $10^{5}$ training samples and with more than $10^{5}$ attributes. The major advantage of SGD is that it can handle large dataset. Here, in this research problem small size of dataset has been used but this approach can be extended this research for up to $10^{5}$ features.

\section{MODEL EVALUATING}

Result of three different models are compared. The confusion matrix has been used as a performance metric. Table 2 describes the structure of confusion matrix. The formula for accuracy of model is shown in eq. 4. Table 3, 4 and 5 are confusion matrices of Model A, B and C and Table 6 shows the accuracy score of three models. This table implies that the bi-gram will give more accurate result than unigram. However, in unigram model, number of feature is less than bi-gram model, due to which time in building the model in unigram is less than bi-gram. Here, the accuracy of Model B is higher than Model A because it is trained with Tf-idf. With the increase in feature size (>20000), Model A and B will not provide feasible solutions. To overcome such issues Model C is introduced in this paper and it is trained using SGD, it supports up to $10^{5}$ features (3) for building a model. Thus Model C can be used when the feature size is high, otherwise Model B works well when the feature size is less. 
News Headlines Dataset without Sentiment Score

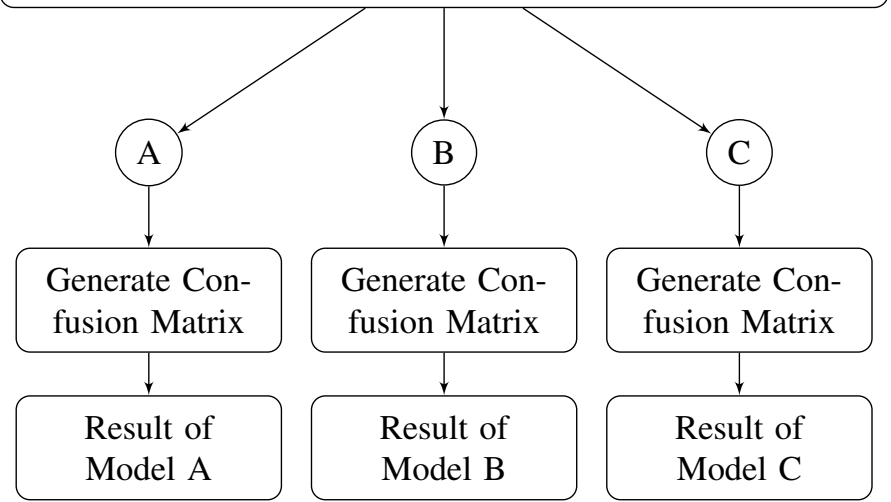

Figure 13. Models evaluation

Table 2. Confusion Matrix

\begin{tabular}{cccc}
\hline & & \multicolumn{2}{c}{ PREDICTED } \\
& & TRUE & FALSE \\
\hline ACTUAL & TRUE & TP & FN \\
& FALSE & FP & TN \\
\hline
\end{tabular}

Accuracy Score $=\frac{T P+T N}{T P+T N+F P+F N} * 100 \%$

Table 3. Model A (Linear SVM) Confusion Matrix

\begin{tabular}{cccc}
\hline \multicolumn{4}{c}{ Unigram Model } \\
\hline & \multicolumn{3}{c}{ PREDICTED } \\
ACTUAL & TRUE & 223 & FALSE \\
& FALSE & 9 & 34 \\
\hline \multirow{4}{*}{ ACTUAL } & Bi-gram Model \\
& TRUE & TRUE & FALSE \\
& FALSE & 4 & 27 \\
\end{tabular}

Table 4. Model B (Linear SVM + Tf-idf) Confusion Matrix

\begin{tabular}{cccc}
\hline \multicolumn{4}{c}{ Unigram Model } \\
\hline & \multicolumn{3}{c}{ PREDICTED } \\
& & TRUE & FALSE \\
ACTUAL & TRUE & 227 & 22 \\
& FALSE & 5 & 41 \\
\hline \multirow{4}{*}{ ACTUAL } & Bi-gram Model \\
& TRUE & TRUE & FALSE \\
& FALSE & 4 & 21 \\
& &
\end{tabular}


Table 5. Model C (SGD) Confusion Matrix

\begin{tabular}{cccc}
\hline \multicolumn{3}{c}{ Unigram Model } \\
\hline & \multicolumn{3}{c}{ PREDICTED } \\
TRUE & FALSE \\
ACTUAL & TRUE & 218 & 34 \\
\hline \multirow{4}{*}{ ACTUAL } & FALSE & 14 & 29 \\
& Bi-gram Model & \\
& & TRUE & FALSE \\
& TRUE & 226 & 29 \\
& FALSE & 6 & 34 \\
\hline
\end{tabular}

Table 6. Accuracy Comparison between Different Models:

\begin{tabular}{lccc}
\hline & Model A (Linear SVM) & Model B (Tf-idf + Linear SVM) & Model C (SGD) \\
\hline Unigram & $87.11 \%$ & $90.84 \%$ & $83.72 \%$ \\
Bi-gram & $89.49 \%$ & $91.52 \%$ & $88.13 \%$ \\
\hline
\end{tabular}

\section{CONCLUSION AND FUTURE SCOPE}

Opinion mining is a vast research domain which can be implemented using Neural Networks, NLP etc. The proposed method is implemented and tested on National/International news headlines of many different regions. The proposed method outperforms existing model. However, the proposed method can not handle sarcasm.

As a future work, based on data accessibility, the proposed method can be implemented on social media data like Twitter, Facebook etc. Also in public sector, the proposed method can be used for the government to analyze impact of a policy in particular region. The proposed method can be altered to handle sarcasm in process I and II in order to increase the accuracy of the model.

\section{REFERENCES}

[1] D. Dor, “On newspaper headlines as relevance optimizers," Journal of Pragmatics, vol. 35, no. 5, pp. 695 - 721, 2003. [Online]. Available: http://www.sciencedirect.com/science/article/pii/S0378216602001340

[2] C. D. Manning, M. Surdeanu, J. Bauer, J. Finkel, S. J. Bethard, and D. McClosky, "The Stanford CoreNLP natural language processing toolkit," in Association for Computational Linguistics (ACL) System Demonstrations, 2014, pp. 55-60. [Online]. Available: http://www.aclweb.org/anthology/P/P14/P145010

[3] F. Pedregosa, G. Varoquaux, A. Gramfort, V. Michel, B. Thirion, O. Grisel, M. Blondel, P. Prettenhofer, R. Weiss, V. Dubourg, J. Vanderplas, A. Passos, D. Cournapeau, M. Brucher, M. Perrot, and E. Duchesnay, "Scikit-learn: Machine learning in Python," Journal of Machine Learning Research, vol. 12, pp. 28252830, 2011.

[4] A. Agarwal, V. Sharma, G. Sikka, and R. Dhir, "Opinion mining of news headlines using sentiwordnet," in Colossal Data Analysis and Networking (CDAN), Symposium on. IEEE, 2016, pp. 1-5.

[5] S. Baccianella, A. Esuli, and F. Sebastiani, "Sentiwordnet 3.0: An enhanced lexical resource for sentiment analysis and opinion mining." in LREC, vol. 10, 2010, pp. 2200-2204.

[6] K. Yang, Y. Cai, D. Huang, J. Li, Z. Zhou, and X. Lei, "An effective hybrid model for opinion mining and sentiment analysis," in Big Data and Smart Computing (BigComp), 2017 IEEE International Conference on. IEEE, 2017, pp. 465-466.

[7] S. Rana and A. Singh, "Comparative analysis of sentiment orientation using svm and naive bayes techniques," in Next Generation Computing Technologies (NGCT), 2016 2nd International Conference on. IEEE, 2016, pp. 106-111. 
[8] R. K. Bakshi, N. Kaur, R. Kaur, and G. Kaur, "Opinion mining and sentiment analysis," in Computing for Sustainable Global Development (INDIACom), 2016 3rd International Conference on. IEEE, 2016, pp. 452-455.

[9] S. Aroju, V. Bhargavi, S. Namburi et al., "Opinion mining on indian newspaper quotations," in Signal Processing and Integrated Networks (SPIN), 2016 3rd International Conference on. IEEE, 2016, pp. 749-752.

[10] K. A. Hasan, M. S. Sabuj, and Z. Afrin, "Opinion mining using naïve bayes," in Electrical and Computer Engineering (WIECON-ECE), 2015 IEEE International WIE Conference on. IEEE, 2015, pp. 511-514.

[11] H. Akkineni, P. Lakshmi, and B. V. Babu, "Online crowds opinion-mining it to analyze current trend: A review," International Journal of Electrical and Computer Engineering (IJECE), vol. 5, no. 5, pp. 1180-1187, 2015.

[12] P. Arora, D. Virmani, and P. Kulkarni, "An approach for big data to evolve the auspicious information from cross-domains," International Journal of Electrical and Computer Engineering (IJECE), vol. 7, no. 2, pp. 967-974, 2017.

[13] A. R. Susanti, T. Djatna, and W. A. Kusuma, "Twitter's sentiment analysis on gsm services using multinomial naïve bayes." Telkomnika, vol. 15, no. 3, 2017.

[14] J. Jotheeswaran and Y. Kumaraswamy, "Opinion mining using decision tree based feature selection through manhattan hierarchical cluster measure." Journal of Theoretical \& Applied Information Technology, vol. 58, no. 1, 2013.

[15] C. Bhadane, H. Dalal, and H. Doshi, "Sentiment analysis: measuring opinions," Procedia Computer Science, vol. 45, pp. 808-814, 2015.

[16] J. Kaur and S. Vashisht, "Analysis and indentifying variation in human emotion through data mining," International Journal of Computer Technology and Applications, vol. 3, no. 6, p. 1963, 2012.

[17] I. Smeureanu and C. Bucur, "Applying supervised opinion mining techniques on online user reviews," Informatica economica, vol. 16, no. 2, p. 81, 2012.

[18] K. Gao, H. Xu, and J. Wang, "A rule-based approach to emotion cause detection for chinese micro-blogs," Expert Systems with Applications, vol. 42, no. 9, pp. 4517-4528, 2015.

[19] D. Chen and C. Manning, "A fast and accurate dependency parser using neural networks," in Proceedings of the 2014 conference on empirical methods in natural language processing (EMNLP), 2014, pp. 740 750 . 